\title{
Análisis de factores asociados a la participación en actividades recreativas de la comunidad universitaria del Tecnológico de Costa Rica: una estrategia recreativa
}

\author{
Analysis of factors associated with participation \\ in leisure activities of the university community of \\ Technological Institute de Costa Rica: \\ a recreation strategy
}

\author{
Jorge A. Vega Agüero \\ Instituto Tecnológico de Costa Rica \\ Cartago, Costa Rica \\ edgonaster@gmail.com
}

Recibido: 07-VI-2012 • Aceptado: 10-IX-2012 • Corregido: 03-XII-2012

\begin{abstract}
Resumen: La recreación promueve el bienestar integral de las personas, tal beneficio se percibe a nivel mental, físico, social emocional y espiritual. En el plano universitario, las múltiples responsabilidades académicas y laborales disminuyen la posibilidad de participación en las diferentes actividades recreativas que se planifican.

El objetivo del diagnóstico es determinar los motivos y limitaciones de participación recreativa que percibe la comunidad universitaria del Instituto Tecnológico de Costa Rica (TEC) en su Sede Central.

El estudio cualitativo posee una muestra de 7 personas y el cuantitativo de 980 personas. El análisis de los datos se realizó mediante un grupo focal, dos entrevistas, una correlación de spearman, un análisis Chi-cuadrado y cuatro ANOVA de tres vías.

Los resultados muestran que para la comunidad universitaria TEC, la principal motivación de participación recreativa en las mujeres es el factor dominio de competencia y en los hombres es el factor social. Por su parte, para la comunidad estudiantil fueron el intelectual y el dominio de competencia. En el caso de las limitaciones de participación, para las mujeres es el factor estructural y en la comunidad estudiantil fueron los factores interpersonales y estructurales.
\end{abstract}

\section{Introducción}

El Consejo Nacional de Rectores (CONARE) ha asumido un compromiso con las universidades públicas costarricenses para desarrollar nuevas propuestas, programas y proyectos que favorezcan la salud de la comunidad universitaria, específicamente en el uso del tiempo libre y la recreación. Se ha mencionado que la salud integral es la principal condición del desarrollo humano, es el estado del bienestar ideal y solamente se alcanza cuando existe un equilibrio entre los factores físicos, biológicos, emocionales, mentales, espirituales y sociales, que permiten un adecuado crecimiento y desarrollo en todos los ámbitos de la vida (Barboza, 2010).

La recreación favorece la salud de las personas por medio de los beneficios 
El proceso estratégico de la implementación del presente proyecto inicia dentro de la Feria de la Salud (FS) del TEC, pero pretende llegar a ser permanente, es por ello que considera aspectos como la misión, la visión, los objetivos, el diagnóstico (FODA) y la propuesta.

Palabras claves: Recreación, motivos y limitaciones de participación recreativa, comunidad universitaria y propuesta recreativa.

\begin{abstract}
Leisure promotes the well-being of individuals; such benefit is perceived at the mental, physical, social, emotional and spiritual areas. At the university, multiple academic and work responsibilities diminish the possibility of participation in leisure activities.

The objective of this diagnosis was to determine the motives and constraints of leisure participation perceived by the community of the Technological Institute of Costa Rica (TEC). The qualitative study has a sample of 7 people and the quantitative 980. The data analysis was performed using a focus group, two interviews, a spearman correlation, Chi-square analysis and four three-way ANOVA.

The results show that for the TEC university community, the main motivation for leisure participation in women is the competence-mastery factor, and for men, the social factor. For the student community, on the other hand, the intellectual and the competencemastery factor were the main motivation. In relation to the constraints of leisure participation, the structural factor was the most important for women, and for the student community the interpersonal and structural factors.

The strategic process of this project started within the TEC health fair (FS), but there is intention to make it permanent; therefore it is important to consider aspects such as mission, vision, objectives, diagnosis (SWOT) and proposal.
\end{abstract}

Keywords: Leisure, leisure participation, motives and constraints, the university community and leisure proposal. que aporta, varios autores como por ejemplo Salazar (2007) y Vargas (2009) los han detallado, en términos generales, se perciben desde todas las áreas del ser humano. La participación activa y regular en programas con enfoque recreativo colaboran en la calidad de vida; sin embargo, antes de la formulación de un programa, es fundamental realizar un diagnóstico. Esta será la base sobre la cual se realiza el programa, en este caso son los factores de participación que están relacionadas con las motivaciones y las limitaciones de participación recreativa que percibe la comunidad universitaria del Instituto Tecnológico de Costa Rica (TEC). Los dos objetivos del proyecto están relacionados con el diagnóstico y la propuesta de actividades a partir de los resultados, seguidamente se detallan:

1. Analizar los factores que influyen en la participación en actividades recreativas en la comunidad universitaria de la Sede Central del TEC.

2. Contribuir con la promoción y prevención de la salud integral de la comunidad universitaria del TEC en la Sede Central mediante la práctica de actividades recreativas.

\section{Actividades recreativas en el Instituto Tecnológico de Costa Rica (TEC)}

\section{El TEC}

El TEC es una institución de educación superior universitaria, fundada el 10 de junio de 1971. Inicia su funcionamiento en el año de 1973, con su Sede Central ubicada en la provincia de Cartago. Esta entidad pública está conformada por cuatro entes muy importantes: Asamblea Institucional, Consejo Institucional, Rector 
y cuatro Vicerrectorías, las cuales poseen a su cargo Escuelas y Departamentos entre los que se destacan, para el presente proyecto, el Departamento de Salud y la Escuela Cultura y Deporte según Mora (2002).

El Departamento de Trabajo Social y Salud desarrolla programas de atención socioeconómica y de salud dirigidos a la comunidad universitaria. Su fin principal es favorecer la atracción, la permanencia y la conclusión exitosa de la comunidad estudiantil en las carreras del TEC (ITCR, 2010a).

La Escuela de Cultura y Deporte tiene como responsabilidad académica impartir cursos correspondientes a programas docentes, a su vez, ejecutar programas de extensión e investigación. Su enfoque parte de la importancia que tiene el arte, la acción social, el deporte y la recreación en el desarrollo de las personas, con la convicción de que la formación e impulso de los valores humanos es imprescindible para el éxito profesional (ITCR, 2010b).

\section{Recreación}

El concepto de recreación es amplio, prueba de ello son las múltiples definiciones que se pueden encontrar. La recreación consiste en cualquier actividad que se realiza en el tiempo libre, por ejemplo realizar deportes, leer o acampar (Hsin-jung, 2010; Waichman, 2009; Vargas 2009 y Árraga y Sánchez, 2007). Para Waichman (2009) y Vargas (2009) son actividades que deben tener un sentido positivo y constructivo cuyo único fin es la diversión, además una forma de compensación al cansancio producido por tareas cotidianas. Por su parte, Vargas (2009), la define como actividades socialmente aceptables en las que el participante voluntariamente se involucra en acciones libres de las cuales se deriva una satisfacción inmediata. Finalmente, Árraga y Sánchez (2007) anotan que la recreación consiste en actividades educativas y estructuradas con procesos pautados que favorecen la autonomía de las personas.
Con base en la información anterior, puede definirse la recreación como aquellas actividades propias de cada individuo que se realizan en el tiempo libre después de cumplir las responsabilidades laborales, de estudio o ambas, y que favorecen el bienestar de las personas.

Como se ha mencionado, un elemento propio de la recreación es el tiempo libre, este es un tiempo fuera de las horas laborales y académicas. Esta definición es muy simple y limitada, puesto que el tiempo libre es más que eso como se muestra a continuación. Por ejemplo, para Salazar (2007), es la porción de tiempo no dedicada al trabajo, a responsabilidades relacionadas con el trabajo, a formas de cuido personal o a obligaciones familiares y sociales. Para Esqueda y López (2008), es un espacio de tiempo que involucra actividades y prácticas preferidas por cada individuo. En el caso de Brinnitzer (2003), es el tiempo libre de obligaciones, libre para el desarrollo y la autorrealización.

En definitiva, el tiempo libre es un espacio de tiempo fuera de las responsabilidades laborales, académicas y personales, en el cual se eligen y realizan actividades de gusto propio. Además, para que se relacione con la recreación necesita ser utilizado en el bienestar personal por medio de los beneficios que se generan.

\section{Beneficios de la recreación}

A partir de la participación recreativa se perciben sus beneficios en las diferentes áreas del ser humano. En el área física, algunos estudios han destacado los beneficios de la recreación en esta área del ser humano. Según Hass y Sipthorp (2004), algunos programas recreativos en Estados Unidos, como el "MerryMilers", dirigido a personas adultas mayores, cumple con sus principales objetivos: mejora en la salud física, cardiovascular y respiratoria. Además, incrementa la densidad capilar musculo-esquelética, incrementa el 
colesterol bueno (HDL) y disminuye los triglicéridos en sangre (American College of Sports Medicine, 2000).

Además del bienestar físico, el área sociosicológica de las personas también se favorece con la recreación, esta es reconocida por profesionales que investigan y afirman que un programa recreativo genera bienestar personal y felicidad, mejora el autoconcepto y la autoeficacia (Carruthers, 2005).

Según Long et al. (1999); American College of Sports Medicine (2000); Kim (2009) y Valerín y Sánchez (2004), la disminución de la ansiedad y depresión, sobre todo para el aumento en el rendimiento del trabajo y en las actividades deportivas y recreativas, es producto de la participación en actividades físicas en el tiempo libre. Estos autores indican que este tipo de actividades influyen positivamente en el estado de ánimo y ayudan a reducir el estrés, aspectos fundamentales para evitar enfermedades.

Otras áreas beneficiadas por la recreación son: la cognitiva y espiritual, ambas relacionadas indirectamente con la salud de una persona. Norling, Sibthorp, Suchy, Hannon y Ruddell (2010) indican que realizar actividad física de manera recreativa a cierta intensidad influye positivamente en la atención de las personas, cuyo rango de edad es de 18 y 30 años. Por su parte, Norling y Sibthorp (2006) afirman que el humor, la satisfacción personal, el pensamiento creativo, la habilidad de resolver problemas, el incremento de la retención en la comunidad trabajadora y estudiantil son elementos que se favorecen al ser parte de un programa recreativo.

Los programas recreativos tienen un punto de partida, el cual es el diagnóstico. Este recauda información que permitirá determinar la extensión y los tipos de necesidades de las personas que participan en el programa, seguidamente la información obtenida es analizada y ordenada por prioridad para determinar cuáles pueden ser satisfechas por la organización (Salazar, 2010). En este sentido, valorar los factores (motivos y limitaciones) que intervienen en la participación en actividades recreativas son la base de un programa.

\section{Factores de participación recreativa}

En el caso de los motivos de participación para que exista una acción, es necesario encontrar un motivo, en algún momento de la vida las personas han sentido la necesidad de hacer algo o por el contrario no hacerlo, ejemplo de algunas actividades con estas características son: leer, estudiar, comer, pasear, bailar y jugar.

La hipótesis jerárquica de necesidades explica que dentro de todo ser humano existe un escalafón de las siguientes cinco necesidades que se mencionan de forma ascendente: las fisiológicas (el hambre, la sed y otras necesidades corporales), de seguridad (la seguridad y protección de daños físicos y emocionales), sociales (afectos, aceptación y amistad), de estima (respeto a uno mismo, la autonomía, los logros, status y el reconocimiento), de autorrealización (el crecimiento, alcanzar el potencial de uno y la autosatisfacción). Dicha teoría menciona que en la medida que se satisface cada una de estas necesidades en lo sustancial, el siguiente nivel de necesidades se vuelve prioridad (Robbins, 2004).

Se ha encontrado una relación positiva entre la motivación y la participación en actividades recreativas (Hsieh, 1998). Por ejemplo, en un grupo de adultos mayores en Taipei y Kaohsiung, Taiwán, las motivaciones para ser parte de un programa recreativo se dirigen a evitar situaciones estimulantes como buscar la soledad, el descanso y las condiciones de calma (Wang, 2008). Otra investigación en colegiales que practicaban artes marciales los motivaba el factor intelectual, por ejemplo: generar más conocimientos, explorar nuevas ideas, ser creativos entre otros elementos (Chiu, 2008). 
En Costa Rica las investigaciones han sido limitadas en temas de la motivación y la recreación, estas se han enfocado al aspecto físico, en el caso de adultos mayores que participan en actividades físico-recreativas, cuyos motivos de participación más destacados son la autorrealización personal, la búsqueda de estatus y la salud, el segundo factor tiene que ver con actividades que involucren acción, emociones y liberación de energía (Sancho, 2007).

Otro factor que incide sobre la participación en actividades recreativas en la actualidad tiene que ver con las limitaciones para la práctica de actividades recreativas, estas son barreras, impedimentos o pretextos que se buscan para no ser parte de un programa recreativo, negándose así la posibilidad de sentirse bien. Para algunos autores, las limitaciones recreativas son las razones percibidas o experimentadas que inhiben o prohíben la participación, por ejemplo en países desarrollados el obstáculo más frecuente de participación es la falta de tiempo (Han, 2004; Hawkins, Peng, Hsieh y Eklund, 1999).

Para Reichert, Barros, Domínguez y Hallal (2007), hay una relación fuerte entre el número limitaciones percibidas y la participación en actividades deportivas con características recreativas. Este estudio realizado a personas de 20 años o más, en Inglaterra, revela que las principales limitaciones son la baja motivación y la falta de dinero, con base en estos resultados se sugieren estrategias hacia la participación en este tipo de actividades.

En Costa Rica, Sancho (2007) destacó en un grupo de personas adultas mayores que participan en actividades físicorecreativas, que para el $84,7 \%$ de los participantes no existen barreras o limitaciones para realizar actividad física de manera recreativa, sin embargo, el restante 15,3\% considera que la única barrera o limitación son las enfermedades, seguidas por la pereza y la falta de tiempo.

\section{Estrategias organizacionales}

Una vez obtenidos los resultados de un diagnóstico se planea una estrategia de aplicación, la cual es un plan para interactuar con el entorno competitivo que permita lograr los objetivos organizacionales, las estrategias pueden incorporar varias técnicas para el logro de los objetivos y estas se tratan de desarrollar de manera congruente con el ambiente exterior (Daft, 2000).

La misión y la visión de una empresa son elementos filosóficos muy generales cuyo propósito es orientar a la organización. Para poder llegar a ellos es fundamental redactar metas y objetivos que son componentes más específicos. Los objetivos, como apunta Daft (2000), se derivan de la misión y visión, designan los fines que se buscan mediante los procedimientos de operación actuales, en ellos se describen resultados medibles. Los objetivos brindan un sentido de dirección a quienes participan en la organización, ayudan a motivar a los participantes, también son lineamientos para la toma de decisiones de los empleados, además son una norma para la evaluación.

Finalmente, el análisis FODA es un instrumento de evaluación del cual se vale una organización para diagnosticar la situación que vive en la actualidad y así de esta manera tomar decisiones con los objetivos planteados. En este modelo las fortalezas y las debilidades son análisis internos y esto favorece el actuar directamente sobre ellas. Pero las amenazas y las oportunidades son externas, lo que suele ser difícil modificarlas (Ascencio, 2004).

Este instrumento permite valorar el ambiente universitario sobre la situación actual en cuanto a la recreación en la comunidad y así formular planteamientos con más objetividad.

\section{Recreación universitaria}

En el más reciente Plan Nacional de la Educación Superior Universitaria 
Estatal 2006-2010 se plantea como principio la formación humanística cuyo enfoque es el compromiso con la formación integral de la persona, en todos sus alcances y dimensiones (Consejo Nacional de Rectores, 2005).

En la universidad, la recreación se enfoca en oportunidades de convivencia grupal, mediante torneos, festivales $\mathrm{y}$ actividades recreativas, pero en muchas ocasiones estos esfuerzos son aislados y se le delega la responsabilidad a una sola persona para desarrollar todos los programas recreativos, por lo tanto se hace necesario unir esfuerzos con otras instituciones o departamentos para que el impacto sea mayor (Camacho, 2007; Fouce y Gómez, 2003).

Es por ello que estas instituciones tienen la obligación de intervenir en la enseñanza del uso adecuado del tiempo libre por medio de programas que impliquen actividades recreativas como una manera de cultivar adecuados hábitos de vida para que sean acompañados durante su vida académica y laboral, un ejemplo de ello es el formato Feria de la Salud (FS).

A nivel universitario la FS es la oportunidad de promover estilos de vida saludable por medio de la recreación, ya que estas poseen ciertas características que la hacen atractiva al público, por ello son tan visitadas por las personas, algunas de las principales son: fácil acceso, educan sobre la salud de las personas, se evalúa el estado médico, es coordinado por personas de la comunidad y son de bajo costo, estas facilidades podrían ser aprovechadas por la recreación para desarrollar programas en este nivel (Ness, Gurney y Ice. 2003).

\section{Justificación}

Por lo tanto, y con base en toda la información recolectada, el proyecto se justifica a partir de que: a) El Consejo Nacional de Rectores recomienda trabajar en el área de la salud integral.

b) La recreación mediante sus beneficios promueve la salud integral.

c) Las estrategias y los programas en el campo de la salud deben ser novedosos.

d) Las FS deben fortalecer sus programas con la recreación.

e) La participación en un programa recreativo posee un mayor impacto cuando se determinan los factores que intervienen en la participación.

f) El TEC carece de un estudio en factores asociados a la participación recreativa.

\section{Material y Métodos}

La investigación de este estudio es transversal descriptiva. Según Ortiz (2004), estos diseños describen las relaciones entre dos o más variables.

\section{Población y muestra}

Se contó con dos grandes grupos de participantes, cada uno correspondiente a una etapa distinta del diagnóstico:

\section{Etapa uno}

Las personas participantes en esta etapa corresponden al grupo organizador de la FS en el TEC. Son cinco profesionales que laboran en el área de salud de esta institución. En la misma etapa participaron las personas encargadas de coordinar el préstamo y uso de las instalaciones deportivas y culturales del TEC.

\section{Etapa dos}

En la etapa dos participó la comunidad institucional del TEC. Para efectos del presente proyecto se encuestaron 399 
hombres y 234 mujeres que corresponden a la comunidad estudiantil. Además se tomó una muestra de 189 hombres y 158 mujeres en el caso del personal docente y administrativo.

\section{Instrumentos}

El proyecto utilizó los siguientes instrumentos para recolectar la información, cada uno correspondiente a una etapa distinta del diagnóstico:

\section{Entrevista}

En el presente proyecto se utilizó la técnica de entrevista enfocada semiestructurada, la cual según Guardián (2007) permite una conversación orientada hacia un tema en específico, en este caso la FS. Esta técnica permite captar información abundante y básica sobre la situación actual de la infraestructura del TEC. El cuestionario contenía 7 preguntas demográficas y 19 preguntas abiertas. Este instrumento fue validado por dos profesionales con experiencia en el campo de la investigación cualitativa. En primera instancia la Licda. Bettzy Rojas Molina, Licenciada en Psicología y Bachiller en Trabajo Social de la Universidad de Costa Rica quien actualmente trabaja en el Tecnológico de Costa Rica. En segunda instancia la Dra. Jacqueline García Fallas, profesora Catedrática de la Universidad de Costa Rica, además funcionaria del Instituto de Investigación en Educación e Instituto de Investigaciones Filosóficas de dicha universidad y profesora del curso Investigación cualitativa de la maestría en recreación. Lo anterior ayuda a garantizar la consistencia interna del instrumento de medición y los objetivos de este proyecto.

\section{Guía para grupo focal}

El instrumento utilizado en el grupo es una guía de preguntas que se llenó el día de reunión con el grupo de organizadores de la FS. Dicha guía incluye cinco secciones: a) información demográfica (nombre, edad, labor dentro de la organización); b) Ferias de la Salud (organización); c) recreación (percepciones); d) factores que inciden en la recreación (motivos y limitaciones); y e) programas o actividades recreativas (diseño y aplicaciones). De la misma forma, este instrumento fue validado por las dos profesionales antes mencionadas.

\section{Escala de motivos de participación recreativa}

La Escala de Motivación Recreativa (EMR) fue propuesta por Beard y Ragheb (Chiu, 2008). Esta escala mide los motivos de participación en actividades recreativas. El instrumento se divide en cuatro factores que son: las motivaciones intelectuales, las sociales, los dominios de competencia y el factor de evasión de estímulos. Seguidamente se detallan estos factores: a) factor intelectual, puntajes altos en este factor muestran interés de participación en actividades recreativas para aprender, investigar, descubrir, crear e imaginar; b) factor social, puntajes altos en este factor muestran interés de participación por la necesidad de amistades, relaciones sociales y sentimientos de respeto mutuo; c) factor dominio de competencia, puntajes altos en este factor muestran interés de participación por sentido de logro, control y desafío a la competición, y d) factor evasión de estímulos, puntajes altos en este factor muestran interés de participación por la motivación intrínseca de alejarse de la sociedad, estar solo en un lugar tranquilo y pacífico para relajarse.

Hsieh (1998) determinó la confiabilidad alpha Cronbanch de cada una de las categorías: escala total: $\alpha=.90$, intelectuales: $\alpha=.90$, sociales $\alpha=.92$, dominio de la competencia $\alpha=.91 \mathrm{y}$ factor de evasión de estímulos $\alpha=.90$. Esta escala es aceptable debido a que las mediciones mayores a 0.6 en el alpha Cronbanch son aprobadas en estudios preliminares (Kent, 2001). 


\section{Escala de barreras de participación recreativa}

El modelo de Escala de Limitaciones Recreativas (ELR) lo plantearon Crawford, Jackson, Godbey (Alfadhil, 1996). Esta escala mide las barreras que limitan la participación en actividades recreativas. La escala se divide en tres categorías: la intrapersonal, la interpersonal y la estructural. A continuación se detalla cada factor: a) factor intrapersonal, puntajes altos en este factor muestra limitaciones de participación por una condición interna del individuo, es un factor personal psicológico, como el estado de ánimo; b) factor interpersonal, puntajes altos en este factor muestran limitación de participación por la negativa de interacción con otros ya sea la familia, los amigos, los compañeros de trabajo; y c) factor estructural, puntajes altos en este factor muestran limitaciones de participación por situaciones como, pocas oportunidades, costo de las actividades, condiciones ambientales (Han, 2004).

Las versiones originales de los instrumentos EMR y EBR, estaban en inglés. Para validar estos instrumentos fue necesaria su traducción y validación la cual se resume en la Tabla 1.1.

Tabla 1.1

Validez y confiabilidad del EMR y ELR con un día de diferencia

\begin{tabular}{lcc}
\hline \multicolumn{1}{c}{ Factor } & Correlaciones & Alpha Crombach \\
\hline Factor intelectual & $.633(* *)$ & .739 \\
Factor social & $.741(* *)$ & .863 \\
Facto dominio competencia & $.772(* *)$ & .889 \\
Factor evasión estimulo & .137 & .296 \\
Factor intrapersonal & $.509(* *)$ & .760 \\
Factor interpersonal & $.528(* *)$ & .741 \\
Factor estructural & $.443(*)$ & .702 \\
\hline
\end{tabular}

Se puede considerar satisfactorio el resultado del proceso de traducción realizado con respecto a la dimensión "evasiónestímulo", al replicarse el proceso con otra muestra con características similares a la primera, se logró obtener una confiabilidad aceptable cuyo Alpha Crombach fue de alfa $=0,645$.

\section{Procedimientos para analizar la información}

El procedimiento para analizar la información se realizó con ayuda los instrumentos: la entrevista, la guía para el grupo focal, el EMR y el ELR.

\section{Entrevista}

Para el análisis de las entrevistas, se imprimieron sus transcripciones, se analizaron las respuestas de las personas coordinadoras de instalaciones y se identificaron los elementos principales.

\section{Guía para grupo focal}

Para el análisis de la información suministrada, se tabularon de manera exacta y en resumen cada una de las respuestas del grupo organizador de la FS, además se identificaron los principales aportes.

\section{El EMR y ELR}

La información obtenida se procesó en el paquete estadístico para las ciencias 
sociales (SPSS versión 15), un programa de computadora para Windows. Las variables dependientes son los factores que se ven influenciados en la participación de actividades recreativas, factores motivacionales y limitaciones recreativas. Por su parte, las variables independientes en este estudio son el sexo, la población y el lugar en el que se realiza la actividad recreativa, dentro de la institución o fuera de ella.

\section{Los siguientes fueron los métodos utilizados:}

Se aplicó una correlación de spearman para el proceso de análisis de calidad (confiabilidad y validez concurrente) de las traducciones del EMR y el EBR.

La Chi-cuadrado es una tabla de contingencia que aporta información conjunta de dos o más variables mostrando las respuestas de una en función de la otra, indicando el valor que toma la primera variable cuando la segunda tiene un determinado valor (Díaz, 2009). En este caso en particular se determinó la relación entre las categorías del sexo (hombre y mujer), el rol universitario (comunidad estudiantil y personal docente y administrativo) y el lugar en el que practican las actividades recreativas (dentro o fuera del TEC).

Además, para verificar la influencia de las variables independientes (sexo y tipo de población) sobre las variables dependientes (motivaciones recreativas y limitaciones recreativas, es decir, las categorías del EMB y ELR), se aplicaron cuatro análisis de varianza de tres factores (ANOVA). En este análisis, se determinan los factores que influyen en la participación (motivaciones o limitaciones), según el tipo de población, el sexo y lugar de participación. Según Álvarez (1995), el análisis de varianza es una técnica estadística que permite entre otras cosas comparar dos o más medidas de forma simultánea. En el modelo de una vía sólo hay una variable independiente, si hubiese dos en el modelo se aplicaría el análisis de varianza de dos vías y así sucesivamente.

\section{Resultados}

\section{Análisis cualitativos}

Según los datos recolectados en el grupo focal, la comunidad universitaria necesita de motivos externos para participar en las actividades de la FS, de lo contrario serían muy pocos quienes se acercan a este tipo de actividades.

Por otra parte, en un análisis en conjunto por parte del comité organizador, se detectaron limitaciones de participación que podrían influir en la participación de la FS y tiene que ver con horarios, cultura administrativa del TEC y poca credibilidad en la FS, que son factores relacionados a la estructura en el TEC.

La discusión del grupo focal consideró que la incorporación de la recreación durante la FS puede ser un factor de motivación para las personas participantes, ya que promueve una variedad de posibilidades aptas para todo público como los son actividades al aire libre, actividades culturales y deportivas. La organización considera de suma importancia incorporar actividades recreativas para la promoción y prevención en los estilos de vida de la comunidad universitaria, estilos de vida con mucho estrés por el estudio y las labores que se planifican, así lo refuerza Salazar (2007).

En el caso de las entrevistas, los componentes principales de participación están relacionados con la autorrealización, este mismo fenómeno se percibe al analizar el grupo focal. Por otra parte, desde las coordinaciones de instalaciones deportivas y culturales se perciben el factor estructural y el intrapersonal, como elementos que inciden en la participación recreativa del TEC.

\section{Análisis cuantitativos}

Un total de 1200 cuestionarios fueron distribuidos en la comunidad institucional, 
de estos 980 fueron válidos y 120 estaban incompletos o no fueron devueltos. La recolección de los datos se desarrolló en el segundo semestre del 2011 en la comunidad universitaria del TEC.

La muestra está distribuida en dos grandes grupos, el sexo y el rol universitario, los cuales se distribuyen de la siguiente manera: en función del sexo la muestra está conformada por un $40 \%(\mathrm{n}=392)$ de mujeres y un $60 \%$ de hombres $(n=588)$. En relación con el rol universitario el 64,6 $\%(n=633)$ corresponde a la comunidad estudiantil y el $35,4 \%(n=347)$ a personal docente y administrativo.

Seguidamente, se analizó la participación recreativa interna $\left(\mathrm{x}^{2}=3,98 \mathrm{y}\right.$ $\mathrm{p}<0,05)$ al TEC. Por un lado el grupo que mayoritariamente participa actividades recreativas son los hombres. Por otro lado el grupo que en su mayoría no participa en actividades recreativas dentro del TEC es el personal docente y administrativo.

En relación con la participación recreativa externa al TEC $\left(\mathrm{x}^{2}=4,41 \mathrm{y}\right.$ $\mathrm{p}<0,05)$, los grupos analizados que sí participan son las mujeres, las mujeres estudiantes, los hombres estudiantes, los hombres funcionarios, la comunidad estudiantil, el personal docente y administrativo.

Los grupos que no participan, mayoritariamente, en actividades recreativas fuera del TEC son las mujeres estudiantes y las mujeres funcionarias. Por su parte, los hombres son más participativos que las mujeres. Finalmente y en términos generales, se encontraron diferencias significativas entre los grupos a favor de la participación recreativa interna, lo que permite visualizar una participación alta en las actividades que se desarrollen.

Otro objetivo de este proyecto fue determinar la relación entre las personas que conforman la comunidad universitaria y los factores que determinan la participación recreativa. La comunidad universitaria para efecto del análisis se dividió en cuatro grandes grupos: sexo, rol universitario y la participación interna o externa del TEC.
Al considerar la participación interna TEC en actividades recreativas, en función del sexo, el factor más importante para los hombres es social $(\mathrm{F}=8,06, \mathrm{p}<0,05)$, para las mujeres son el dominio de competencia $(\mathrm{F}=15,06, \mathrm{p}<0,05)$ y el factor estructural $(\mathrm{F}=10,51, \mathrm{p}<0,05)$, en la ELR.

En función del rol universitario, los factores más importantes para la comunidad estudiantil son: el intelectual $(\mathrm{F}=10,38, \mathrm{p}<0,05)$ y dominio de competencia $(F=46,85, p<0,05)$, en el caso de EMR, y el interpersonal $(\mathrm{F}=0,66, \mathrm{p}$ $<0,05)$ y estructural $(\mathrm{F}=5,29, \mathrm{p}<0,05)$, en la ELR.

En el caso de la participación interna del TEC, se encontró que en las personas que sí participan, el factor más importante es el dominio de competencia $(\mathrm{F}=6,59, \mathrm{p}$ $<0,05)$. En el caso de la no participación, los factores más importantes son en el ELR, el intrapersonal $(\mathrm{F}=8,18, \mathrm{p}<0,05)$ y el estructural $(\mathrm{F}=11,47, \mathrm{p}<0,05)$.

Finalmente, al considerar la participación recreativa externa del TEC, en función del sexo el factor más importante, en el EMR, para los hombres es el dominio de competencia $(\mathrm{F}=5,04, \mathrm{p}<0,05)$ y el estructural $(\mathrm{F}=6,79, \mathrm{p}<0,05)$ de las ELR, en las mujeres no se presentaron diferencias significativas.

En función del rol universitario, los factores más importantes para la comunidad estudiantil son: el intelectual $(\mathrm{F}=11,79, \mathrm{p}<0,05)$ y dominio de competencia $(\mathrm{F}=45,68, \mathrm{p}<0,05)$, en el caso de EMR, y el interpersonal $(\mathrm{F}=8,36, \mathrm{p}<0,05)$ y estructural $(F=9,11, p<0,05)$, en la ELR. Además se determinó una interacción significativa en los estudiantes que no participan en actividades recreativas en los factores interpersonal $(\mathrm{F}=7,29, \mathrm{p}<0,05)$ y estructural $(\mathrm{F}=6,29, \mathrm{p}<0,05)$.

En el caso de la participación externa del TEC, se determinó que en las personas que sí participan los factores más importante son el intelectual $(\mathrm{F}=6,24, \mathrm{p}<0,05)$ $\mathrm{y}$ el dominio de competencia $(\mathrm{F}=14,12, \mathrm{p}$ $<0,05)$, para el EMR. 


\section{Discusión}

En el presente estudio se determinaron una serie de factores que intervienen en la participación recreativa en la comunidad universitaria del TEC, estos son base de un programa cuya implementación será anual. Los resultados surgen a partir de las técnicas de recolección tanto cualitativas (grupo focal y entrevista) como cuantitativas (Chi cuadrado y ANOVAS).

Seguidamente, se analizó la participación recreativa interna al TEC. Por un lado, el grupo que mayoritariamente participa actividades recreativas son los hombres. Por otro lado, el grupo que en su mayoría no participa en actividades recreativas dentro del TEC es el personal docente y administrativo.

En relación con la participación recreativa externa al TEC, los grupos analizados que sí participan son las mujeres, las mujeres estudiantes, los hombres estudiantes, los hombres funcionarios, la comunidad estudiantil, el personal docente y administrativo.

Los grupos que no participan, mayoritariamente, en actividades recreativas fuera del TEC son las mujeres estudiantes y las mujeres funcionarias. En términos generales, los hombres participan más que las mujeres, además se encontraron diferencias significativas entre los grupos a favor de la participación recreativa interna, lo que permite visualizar una participación alta en las actividades que se organicen.

En el caso de los análisis cualitativos, en el grupo focal se ha determinado que la comunidad universitaria participa en actividades recreativas por motivos externos, estos resultados coinciden con los encontrados por Sancho (2007) y López y Villalobos (2004), en los cuales en personas adultas mayores y mujeres entre 13 y 18 años, les motivan aspectos relacionados con las recompensas en función de la autorrealización. Por su parte, en las entrevistas los componentes principales de participación están relacionados con la autorrealización al igual que el grupo focal.

En el mismo sentido, en el análisis cuantitativo, al considerar la participación interna del TEC en actividades recreativas, en función del sexo, el motivo más importante para los hombres es social y para las mujeres es el dominio de competencia. En función del rol universitario los factores más importantes para la comunidad estudiantil son: el intelectual y dominio de competencia. En el caso de la participación interna del TEC, se encontró que en las personas que sí participan, el factor más importante es el dominio de competencia.

$\mathrm{Al}$ considerar la participación recreativa externa del TEC en actividades recreativas en función del sexo, el factor de motivación más importante para los hombres es el dominio de competencia. En función del rol universitario los factores más importantes para la comunidad estudiantil son: el intelectual y dominio de competencia. En el caso de la participación externa del TEC, se determinó que en las personas que sí participan, los factores más importantes son el intelectual y el dominio de competencia.

Varios estudios han mostrado resultados similares, tal es el caso de Derom (2009) en el cual el principal factor motivador en hombres es el dominio de competencia, por su parte en las mujeres son el intelectual y el domino de competencia (Chiu, 2008).

En segunda instancia, se detectaron limitaciones de participación. Según el grupo focal, las principales inhibiciones tienen que ver con horarios, cultura administrativa del TEC y poca credibilidad en la FS, que son factores relacionados a la estructura en el TEC, así también estudios encuentran estas situaciones con principales limitaciones de participación en diferentes poblaciones (Dunlop, 2006 y Barrantes, 2004). Por otra parte, desde las coordinaciones de instalaciones deportivas y culturales se perciben el factor estructural y el intrapersonal como elementos que inciden en la participación recreativa del TEC. 
Al realizar los análisis cuantitativos en la comunidad cuya participación recreativa es interna en el TEC, en función del sexo, la principal limitación es el factor estructural. En función del rol universitario, son los factores interpersonal y estructural. En el caso de las personas que no participan, los factores más importantes son: el intrapersonal, el interpersonal y el estructural.

Por su parte, al considerar la participación recreativa externa del TEC en actividades recreativas en función del sexo el principal factor de limitación es el estructural. En función al rol universitario, para la comunidad estudiantil son: el interpersonal y estructural. Finalmente, se determinó una interacción significativa en los estudiantes que no participan en actividades recreativas en los factores interpersonal y estructural.

En este mismo orden, se han encontrado estudios cuyos resultados son similares a los obtenidos en el presente, por ejemplo Alfadhil (1996); Nadirova (2000); Dunlop, (2006) en el caso de los factores estructurales. También en pasados estudios se ha determinado como limitantes el factor intrapersonal (Xiong, 2006), interpersonal (Han, 2004).

Es importante considerar que hay factores que condicionan la participación. Según Han (2004) y Gayo-Cal (2006) el nivel de participación depende del tipo de actividad en función del sexo y la edad, por ejemplo, en el caso de los hombres, los deportes y las actividades sociales son las más atractivas y en el grupo de edad entre 29-39 años, se pueden citar uso de la computadora, salir a comer y salir al cine.

En relación con el sexo, Romero (2006) encontró que las actividades preferidas por los hombres estudiantes de la UNED eran fútbol sala, campamentos, baloncesto, ir al cine y los paseos; por su parte, las mujeres estudiantes de la UNED prefieren los aeróbicos, ir al cine, teatro, campamentos y paseos. Por su parte, Han (2004) determinó que las actividades más atractivas para el sexo masculino son las actividades dinámicas y a las mujeres les atraen las actividades pasivas, además varios estudios demuestran que las mujeres presentan más limitantes de participación en comparación con los hombres (Alfadhil, 1996; Han, 2004 y Nadirova, 2000), de la misma manera se comporta la población en la participación recreativa externa del TEC, la mayoría de las mujeres no participan.

Además, según la edad, en el estudio de Han (2004), se encontró que el grupo entre 19-29 años, edad universitaria, participa mayoritariamente en actividades como salir a comer, salir al cine y actividades sociales (fiesta), sin embargo, hay que considerar las limitaciones económicas a nivel universitario, ello explicaría la no participación de las mujeres estudiantes en actividades fuera del TEC. Por otra parte, en las edades laborales entre 29-39 años las actividades preferidas son: usar la computadora, salir a comer y salir al cine. Según Alfadhil (1996), un factor determinante en la participación recreativa en personas mayores de 24 años es el poco tiempo disponible.

Es por esta razón que todos estos factores podrían influir en la participación recreativa interna o externa del TEC y que son base fundamental en la formulación de un programa recreativo en esta institución.

De los anteriores planteamientos se concluye que:

1. La FS del TEC carece de una propuesta objetiva y fundamentada administrativamente en la cual se pueda basar un programa recreativo. Por lo tanto es necesario fundamentar la propuesta recreativa con una estructura organizativa adecuada. Según Salazar (2007), las organizaciones deben de tener una filosofía que guía su actuar en el momento de ofrecer productos o servicios.

2. Según la comisión organizadora de la FS, los motivos externos 
son la principal razón de participación de la comunidad universitaria del TEC. Por otra parte, las principales limitaciones de participación tienen que ver con horarios de la comunidad estudiantil, la cultura administrativa del TEC y la baja credibilidad de población hacia la FS.

3. Es necesario implementar un programa recreativo novedoso y atractivo a la comunidad universitaria que promueva la salud integral de las personas participantes.

4. Las personas que coordinan las instalaciones deportivas y culturales consideran que la comunidad universitaria participa en actividades recreativas con motivaciones externas y en eventos consolidados. Por otra parte, la participación en actividades recreativas está limitada por el bajo apoyo institucional, la escasa divulgación de actividades que se planifican y desarrollan, la baja cultura recreativa en la institución, el bajo interés y el estado de ánimo de las personas.

5. Según los resultados del estudio en la comunidad universitaria del TEC, para los hombres los motivos de participación tienen que ver con los factores sociales y dominio de competencia. En el caso de las mujeres, las actividades cuya motivación de participación más importante fue el factor dominio de competencia.

6. En el caso de la comunidad estudiantil, los factores que motivan la participación recreativa tienen que ver con los factores intelectuales y dominio de competencia.

7. Las limitaciones de participación recreativa más significativa para las mujeres tienen que ver con los factores estructurales.
8. En el caso de las actividades que limitan la partición recreativa de la comunidad estudiantil tienen que ver con el factor interpersonal y estructural.

Finalmente se proponen algunas recomendaciones para futuros estudios similares:

1. Es necesario implementar una estrategia informativa sobre el concepto de recreación, los beneficios y sus implicaciones en el diario vivir, en la comunidad universitaria TEC, de tal forma que permita formar un criterio claro de este concepto.

2. Sería importante, en futuros estudios, considerar los resultados de la validez y confiabilidad de los instrumentos para medir los factores que intervienen en la participación recreativa y profundizar en el análisis de las características psicométricas de este instrumento.

3. Se busca por medio de un programa recreativo crear la cultura recreativa en la comunidad universitaria en función de un estilo de vida saludable.

4. Se deben considerar horarios de participación variados que permitan a todas las personas de la comunidad universitaria tener acceso a alguna actividad que sea de su agrado.

5. Es preciso implementar un trabajo de diagnóstico que permita conocer puntualmente las necesidades e intereses de la comunidad universitaria del TEC.

6. Es imprescindible implementar actividades recreativas para toda la población universitaria, es importante considerar cada elemento que integra la comunidad, ya que en ella se convive diariamente. 


\section{Propuesta}

El siguiente resumen de la propuesta se fundamenta en los resultados del diagnóstico realizado en la comunidad universitaria del TEC, el cual consistió en analizar los factores que influyen en la participación en actividades recreativas de la comunidad universitaria del TEC. La propuesta consiste en un plan piloto como lo recomienda Armida (2005), que pretende contribuir en la promoción y prevención de la salud de la comunidad universitaria del TEC. Para valorar el impacto de las actividades se utilizará inicialmente la FS, que se realiza en una semana. Dicho planteamiento describe 4 subprocesos que comprenden actividades físicas, sociales, multidisciplinarias e intelectuales.

Los objetivos de la propuesta se enfocan en la salud integral, tanto desde la perspectiva de la comunidad universitaria (estudiantes, docentes, personal administrativo) como del nivel individual (fisiológica, intelectual, sociopsicológica). A continuación se presenta el objetivo general y los objetivos específicos de la propuesta recreativa.

\section{Objetivo General}

Promover la salud integral de la comunidad universitaria por medio de actividades recreativas que favorezcan el uso adecuado del tiempo libre.

\section{Objetivos Específicos}

1. Incentivar la actividad física por medio de la recreación para la mejora en la salud física de las personas participantes.

2. Crear espacios para la interacción social en la comunidad universitaria del TEC, mediante actividades recreativas, para mejorar las relaciones entre las personas que la integran.

3. Propiciar la participación en actividades recreativas que impliquen el bienestar psicológico para mejorar la salud mental de las personas.

Las actividades propuestas son:

Tabla 1.1

Resumen de la propuesta recreativa

\begin{tabular}{ll}
\hline \multicolumn{1}{c}{ Proyecto } & \multicolumn{1}{c}{ Propósito } \\
\hline MueveTEC & $\begin{array}{l}\text { Promover la actividad física como un factor importante en la } \\
\text { salud física de la comunidad universitaria del TEC. }\end{array}$ \\
EducaTEC & $\begin{array}{l}\text { Organizar talleres recreativos para personas con alguna limita- } \\
\text { ción física (obesidad, diabetes, hipertensión), para beneficio de } \\
\text { su calidad de vida. }\end{array}$ \\
EspecialTEC & $\begin{array}{l}\text { Integrar a la comunidad externa e interna del TEC a las activi- } \\
\text { dades recreativas planificadas. }\end{array}$ \\
Ludomediodía & Ofrecer juegos de mesa a la comunidad universitaria como \\
& una opción uso adecuado del tiempo libre para mejorar a nivel \\
& cognitivo.
\end{tabular}


La descripción del proyecto comprende: nombre del proyecto, diseño, objetivos, justificación, explicación, contenido, población a la que va dirigida, instalaciones a utilizar, cronograma, materiales, presupuesto, formas de matricularse, medidas de seguridad e higiene, instrumento de evaluación.

Seguidamente se muestra un ejemplo del formato utilizado para desarrollar el proyecto:

\section{Subproyecto 1}

El proyecto uno surge del eje estratégico "Impulsar la participación en actividades recreativas en la comunidad universitaria", resultado del diagnóstico y del análisis FODA que se desarrolló.

Nombre: MueveTEC

Diseño:

- Tipo de actividad: Actividad física y deporte.

- Formato: Grupos con intereses sociales y competencia.

\section{Objetivos}

\section{Objetivo General}

Por medio de actividades recreativas, el Comité Organizador pretende:

1. Conceptualizar la actividad física como un factor importante en la salud de la comunidad universitaria del TEC.

\section{Objetivos Especificos}

Con la participación en las actividades recreativas las personas participantes podrán:

1. Reconocer la actividad física como un elemento importante en la organización del tiempo libre.

2. Experimentar sensaciones de bienestar físico después de la actividad recreativa realizada.

3. Inferir los beneficios físicos que la actividad física genera en las personas que la practican.
4. Implementar de forma permanente este proyecto en el Tecnológico de Costa Rica.

Explicación del proyecto. El proyecto MueveTEC consiste en actividades bailables grupales y torneos de futbol siete capaces de producir un gasto calórico considerable para las personas participantes. En el caso particular de actividades bailables grupales se pueden mencionar: la danza aeróbica, el baile latino, los pilates, entre otros. Finalmente el torneo de futbol siete se jugará en el campo de fútbol, con la participación de la mayor cantidad de equipos.

Justificación. Este tipo de actividad se focaliza en la salud física. En este sentido se han encontrado estudios que demuestran que jugar fútbol de manera recreativa en 12 semanas los jugadores perdieron 12 libras de grasa, además los jugadores ganaron 3.7 libras de músculo, redujo colesterol LDL (Parks and Recreation, 2007 y Blatter, 2010), además el fútbol motiva la interacción social y facilita la adherencia a un estilo de vida activo (Blatter, 2010).

Finalmente, parte de las actividades rítmicas aeróbicas han encontrado beneficios físicos en la resistencia aeróbica, incremento de la fuerza y la masa muscular; por otra parte, se percibe un mejor estado físico en general en lo psicológico además de un bienestar general (Schiffer et. al, 2009). En otro estudio (Hos, 2005), se encontró que las actividades rítmicas aeróbicas influyen sobre la propia imagen en las mujeres, no solo en la imagen corporal sino también en la social y moral, además mejora la confianza y la satisfacción.

Contenidos. Tiempo libre, salud integral, el ejercicio físico, la convivencia social, calidad de vida y el bienestar personal.

Población. El proyecto MueveTEC está dirigido a todas las personas que conforman la comunidad universitaria del TEC: estudiantes, personal docente y administrativo.

Número de grupos. En las actividades bailables seis grupos, cuyo nivel 
oscila entre principiante e intermedio. Por grupo se pueden matricular mínimo 20 personas y máximo 30. En el caso del torneo de fútbol 7 , la participación será de mínimo cuatro equipos y máximo ocho.

Instalaciones. Gimnasio Armando Vásquez y campo de fútbol del TEC.

Materiales. Árbitros, balones de fútbol, campo de fútbol, colchonetas, gimnasio Armando Vásquez, instructor, sonido ambiente. Además, esparadrapo, gasa, paletas alcohol.

Matrícula. La actividad se desarrollará en el Gimnasio Armando Vásquez del TEC y campo de fútbol, con capacidad para 150 personas y 8 equipos de futbol 7. La matrícula será vía digital en "google docs" y las primeras 150 personas y los primeros 8 equipos, sean estudiantes, personal docente y administrativo, matriculados serán notificados con su participación. En el caso de que los recursos sean insuficientes, se buscará patrocinio fuera de la institución que ayude a solventar la actividad.

\section{Medidas de seguridad}

1. Toda persona participante debe aceptar las condiciones del reglamento de uso de instalaciones.

2. El área de salud tendrá un puesto para atender emergencias dentro del gimnasio y cerca del campo de fútbol.

3. Habrá médicos del área de salud presentes.

4. Habrá extinguidores de incendio a mano y en buenas condiciones.

5. Se tendrán a mano los números de teléfono de las instituciones que podrían ayudar en caso de emergencia.

6. Saber dónde está el hidrante más cercano a las instalaciones.

7. Indicar por escrito cuáles son las zonas de seguridad existentes en el lugar, en caso de temblor.

8. Indicar por escrito dónde queda el refugio en caso de tornado.
9. Indicar dónde quedan los servicios sanitarios y las duchas en el gimnasio.

10. Solicitar a cada persona un paño personal, para secar el sudor.

11. Solicitar a cada persona una adecuada hidratación antes, durante y después de la actividad.

\section{Instrumento para evaluar}

Se incluyó una escala por cada objetivo específico, en este caso en particular la subescala 5 de la escala de satisfacción recreativa, la cual según Wu (2010) valora la dimensión fisiológica de satisfacción recreativa. Puntajes altos en esta subescala muestran que las personas alcanzan un estado de bienestar físico por medio de la actividad recreativa.

\section{Referencias}

Alfadhil, A. (1996). University students' perception of constraints to participation in recreational sports. (Tesis de Doctorado de Filosofía sin publicar). Michigan State University, United States.

Alvarez, R. (1995). Estadística multivariante y no paramétrica con SPSS. Madrid, España: Díaz de Santos, S.A.

American College of Sports Medicine. (2000). ACSM's guidelines for exercise testing and prescription (6ta. ed.). Philadelphia, United States: Editorial Johnson.

Armida, A. (2005). Plan de negocios como estrategia competitiva para el campamento Tomacoco (Tesis de Licenciatura en Administración de Empresas sin publicar). Universidad de las Américas, Puebla, México.

Árraga M. y Sánchez, M. (2007). "Recreación y calidad de vida en adultos mayores que viven en instituciones geriátricas y en sus hogares". 
Un estudio comparativo. Cuaderno Venezolano de Sociología, 16(4), 737 756. Recuperado de: https://sibdi.ucr. ac.cr/http://web.ebscohost.com/ehost/ pdfviewer/pdfviewer?sid=debac8a532bc-48f7-924d-4cb5bde51f6a\%40se ssionmgr $11 \&$ vid $=7 \&$ hid $=12$

Ascencio, M. (2004). Simulador internacional de negocios de la Universidad Carnegie Mellon (Tesis de Licenciatura en Administración de Empresas sin publicar). Universidad de las Américas, Puebla, México.

Barboza, Y.V. (2010). "Nutrición esencia de la salud integral". Anales Venezolanos de Nutrición, 23(1), 50-53. Recuperado de: https://sibdi. ucr.ac.cr/http://web.ebscohost.com/ ehost/pdfviewer/pdfviewer?vid=7\& $\mathrm{hid}=12 \& \operatorname{sid}=$ debac $8 \mathrm{a} 5-32 \mathrm{bc}-48 \mathrm{f} 7$ 924d-4cb5bde51f6a\%40sessionmgr11

Barrantes, J. (2004). Factores motivacionales que influyen en la deserción de la mujer de la práctica deportiva (baloncesto y voleibol, I división durante el periodo comprendido entre 1999 y 2001. (Tesis de Licenciatura en Ciencias del Deporte con énfasis en rendimiento sin publicar). Universidad Nacional, Heredia, Costa Rica.

Blatter, J. (2010). "Football for healthprevention is better than cure". Scandinavian Journal of Medicine and Science in Sports, 20(1), 5-5. doi: 10.1111/j.I600.2010.01114x

Brinnitzer, E. (2003). "Adolescencia, pobreza y tiempo libre en mujeres y varones". Revista de Estudios de la Mujer, 2(8), 221-244. Recuperado de: https:// sibdi.ucr.ac.cr/http://web.ebscohost. com/ehost/pdfviewer/pdfviewer?vid= 8\&hid=12\&sid=debac8a5-32bc-48f7924d-4cb5bde51f6a\%40sessionmgr11

Camacho, J. M. (2007). Efecto de un programa recreativo en la población estudiantil de primer ingreso del año 2006, de residencias de la Universidad de Costa Rica (Tesis de
Maestría en Recreación sin publicar). Universidad de Costa Rica, San José, Costa Rica.

Carruthers, C., y Hood, C. (2005). "The power of positive psychology: The paradigm shifts from problem-solving to optimism". Park and Recreation, 40(10), 30-37. Consultado en: https://sibdi. ucr.ac.cr/http://web.ebscohost.com/ ehost/pdfviewer/pdfviewer?vid=10 \&hid=12\&sid=debac8a5-32bc-48f 7 924d-4cb5bde51f6a\%40sessionmgr11

Chiu, W. H. (2008). A study of motivation, satisfaction, and constraints of collegiate chinese martial arts athletes engaged in leisure activities in Taiwan (Tesis de Doctorado en Administración Deportiva sin publicar). United States Sports Academy, United States.

Consejo Nacional de Rectores. (2005). Marco estratégico de acción para el área de vida estudiantil en el Consejo Nacional de Rectores. San José, Costa Rica: CONARE-OPES.

Daft, R. (2000). Teoría y diseño organizacional (4ta.ed.). México D.F.: Editorial Thomson.

Derom, I. (2009). Identity and motives of participants at cause-related sport events (Tesis de Maestría del Movimiento Humano sin publicar). University of Windsor, Canada.

Díaz, V. (2009). Análisis de datos de encuestas. Barcelona, España: Editorial UOC.

Dunlop, P. (2006). Active employees! Exploring the relationship between job characteristics, perceived constraints an participation leisure-time physical activity (Tesis de Maestría en Recreacion sin publicar). North Carolina University, United States.

Esqueda, S., y López, S. (2008). "El ocio de los jóvenes: La gran oportunidad". Debates Instituto de Estudios Superiores de Administración, 13(2), 25-30. Recuperado de: https://sibdi.ucr.ac.cr/http://web. 
ebscohost.com/ehost/pdfviewer/ pdfviewer?sid=debac8a5-32bc-48f7924d-4cb5bde51f6a\%40sessionmgr11 \&vid=13\&hid=12

Fouce, J., y Gómez, E. (2003). "El programa de ocio nocturno juvenil Torrejón a Tope". Intervención Psicosocial, 13(2), 247-260. Recuperado de: http://www. psicopol.unsl.edu.ar/mayo2007_ nota5.pdf

Gayo-Cal, M. (2006). "Leisure and participation in Britain". Cultural Trends, 15(2/3), 175-192. doi:10.1080/09548960600713015

Guardián, A. (2007). Coordinación educativa y cultural Centroamericana (CECC) agencia Española de cooperación. San José, Costa Rica: Editorial Print Center.

Han, W. J. (2004). Leisure participation and constraints: The case of Korean Americans (Tesis de Doctorado en Filosofía sin publicar). The Pennsylvania State University, United States.

Hass, C., y Sipthorp, J. (2004). "Making the benefits last". Parks and Recreation, 39(6), 32-34. Recuperado de: https://sibdi.ucr.ac.cr/http://web. ebscohost.com/ehost/pdfviewer/ pdfviewer?vid $=23$

Hawkins, B., Peng, J., Hsieh, C-M. y Eklund, S. (1999). "Leisure constraints: A replication and extension of construct development". Leisure Sciences, 21(3), 179-192. doi: $10.1080 / 014904099273066$

Hos, A. (2005). "The effects of guided systematic aerobics dance programme on the self- esteem of adults". Kinesiology,37(2), 141-150. Recuperado de: https://sibdi.ucr. ac.cr/http://web.ebscohost.com/ehost/ pdfviewer/pdfviewer?sid=c9bc9578fed3-4673-a4a089506efae1c\%40sessi onmgr $112 \&$ vid=7\&hid $=110$

Hsieh, C. M. (1998). Leisure attitude, participation and satisfaction: Test of model of leisure behavior. (Tesis de
Doctorado en Filosofía sin publicar). School of Health, Physical Education and Recreation, Indiana University, United States

Hsin-jung, S. (2010). An analysis of recreational sport programs for boys and girls in ages 12 to 14 in Taoyuan city, Taiwan (Tesis de Doctorado en Educación de Gestión del Deporte sin publicar). Sports Academy de Alabama, United States

Instituto Tecnológico de Costa Rica. (2010a). Departamento de Trabajo Social y Salud. Cartago, Costa Rica. Recuperado de: http://www.tec. ac.cr/sitios/Vicerrectoria/viesa/tss/ Paginas/default.aspx

Instituto Tecnológico de Costa Rica. (2010b). Escuela de Cultura y Deporte. Cartago, Costa Rica. Recuperado de: http://www.tec.ac.cr/sitios/Docencia/ culturaydeporte/Paginas/default. aspxInstituto

Kent, R. (2001). Data construction and data analysis for survey research. New York, United States: Editorial Palgrave.

Kim, B. (2009). A conceptual framework for leisure and subjective well-being (Tesis de Doctorado en Recreación sin publicar).School of Health, Physical Education and Recreation, Indiana University, United States.

Long, B., Wooten, W., Patrik. K., Caltas, K., Sharper, D., y Sallir, J. (1999). Proyecto muévete. Manual del personal de salud. Evaluación y orientación médica referentes a la actividad fisca. Manuscrito sin publicar.

López, D. y Villalobos, L. (2004). Determinación y comparación de los factores motivacionales que inciden en la práctica de la gimnasia en atletas que comprenden edades entre 13 y 18 años de edad, participantes en el tercer campeonato Centroamericano de Gimnasia, 2003 (Tesis de Licenciatura en Ciencias del Deporte con énfasis en rendimiento y salud, 
sin publicar). Universidad Nacional, Costa Rica.

Mora, M. (2002). Impacto de los jubilados en la Vicerrectoría de Docencia del ITCR, durante los años 2000 al 2002 y de la proyección para el periodo 2002 al 2005. (Tesis para optar por el grado de Licenciatura en Recursos Humanos sin publicar). Instituto Tecnológico de Costa Rica, Costa Rica.

Nadirova, A. (2000). Understanding leisure decision-making: An integrated analysis of recreation participation, anticipated leisure benefits, environmental attitudes, leisure constraints, and constraints negotiation (Tesis de Doctorado en Filosofía sin publicar). Department of Earth an Atmospheric Sciences. Alberta University, Canadá.

Ness, K., Gurney, J., y Ice, G. (2003). "Screening, education, and associated behavioral responses to reduce risk for falls among people over age 65 years attending a community health fair". Physical Therapy, 83(7), 631-637. Recuperado de: http://ptjournal.apta.org/cgi/reprint/83/7/631

Norling, J. C. y Sibthorp, J. (2006). "Mental restoration and recreation taking time to visit a park may leave you more relaxed". Parks and Recreation, 41(3), 30-35. Recuperado de: http://www.thefreelibrary.com/ esearch+update:+mental+restoration +and+recreation:+taking+time+to... -a0143828188

Norling, J. C., Sibthorp, J., Suchy, Y., Hannon. J. y Ruddell. E. (2010). "The benefit of recreational physical activity to restore attentional fatigue: The effects of running intensity level on attention scores". Journal of Leisure Research, 42(1), 135-152. Recuperado de: https://sibdi.ucr.ac.cr/http:// web.ebscohost.com/ehost/pdfviewer/pdfviewer?vid= $42 \&$ hid $=1$
$0 \& \operatorname{sid}=$ debac $8 \mathrm{a} 5-32 \mathrm{bc}-48 \mathrm{f} 7-924 \mathrm{~d}-$ 4cb5bde51f6a\%40sessionmgr11

Ortiz, G. (2004). Diccionario de metodología de la investigación científica. México D.F., México: Editorial Limusa.

Parks and Recreation. (2007). "Soccer Provides Better Exercise". Parks and Recreation, 42(11), 23-23. Recuperado de:https://sibdi.ucr. ac.cr/http://web.ebscohost.com/ehost/ pdfviewer/pdfviewer?sid=c9bc9578fed3-4673-a4a09506efae1c\%40sessio nmgr $112 \&$ vid $=5 \&$ hid $=110$

Reichert, F., Barros, A., D omínguez, M., y Hallal, P. (2007). "The role of perceived personal barriers to engagement in leisure-time physical activity". American Journal of Public Health, 97(3), 515-519. doi:10.2105/ AJPH.2005.070144

Robbins, S. (2004). Comportamiento organizacional (10a. ed.). Atlacomulco, México: Editorial Prentice Hall.

Romero, C. (2006). Propuesta organizacional del área de recreación para la Universidad Estatal a Distancia (Tesis de Magíster en Recreación sin publicar). Universidad de Costa Rica, Costa Rica.

Schiffer, T., Kleinert, J., Sperlich, B., Schult, S. y Struder, H. (2009). "Effects of aerobic dance and fitness programme on physiological and psychological performance in men and women". International Journal of Fitness, 5(2), 37-46. Recuperado de: https://sibdi.ucr.ac.cr/http://web. ebscohost.com/ehost/pdfviewer/ pdfviewer?sid=c9bc9578-fed3-4673a4a0-c89506efae1c\%40sessionmgr11 $2 \&$ vid $=5 \&$ hid $=110$

Salazar, C. G. (2010). Antología del cursos PF-4904 Programación en la recreación. Maestría Profesional en Recreación, Universidad de Costa Rica. San José, Costa Rica.

Salazar, C. G. (2007). Recreación.San José, Costa Rica: Editorial UCR. 
Sancho, J. (2007). Factores motivacionales hacia la actividad física en personas adultas Mayores (Tesis de Licenciatura en Ciencias del Deporte con énfasis en salud, sin publicar). Universidad Nacional, Costa Rica.

Valerín, J., y Sánchez, D. (2004). Efecto de un programa de actividad físicarecreativa en el autoconcepto físico $y$ general, la práctica de actividad física en el tiempo libre y la autoeficacia para la práctica de actividad física de adolescentes de un colegio urbano-marginal. (Tesis de licenciatura con énfasis en salud sin publicar). Universidad Nacional. Heredia, Costa Rica.

Vargas, G. (2009). "Estudio histórico- geográfico de la recreación y el turismo en Costa Rica, 1840-1986". Revista Geográfica, 1(145), 89-120, 32. Recuperado de: https://sibdi.ucr. ac.cr/http://web.ebscohost.com/ehost/ pdfviewer/pdfviewer?vid=48\&hid= $10 \&$ sid=debac 8 a $5-32$ bc $-48 f 7-924 d-$ 4cb5bde51f6a\%40sessionmgr11

Waichman, P. (2009). “Cuál recreación para América Latina?”. Cuaderno
Venezolano de Sociología, 18(1), 101108. Recuperado de: https://sibdi.ucr. ac.cr/http://web.ebscohost.com/ehost/ pdfviewer/pdfviewer?vid=50\&hid= $10 \&$ sid $=$ debac 8 a $5-32$ bc $-48 f 7-924 d$ 4cb5bde51f6a\%40sessionmgr11

Wang, C. C. (2008). Leisure participation, leisure motivation, and life satisfaction for elders in public senior resident homes in Taiwan (Tesis de Doctorado en Filosofía sin publicar). University of the Incarnate Word, San Antonio, Texas, United States.

$\mathrm{Wu}$, C. H. (2010). The relationship among leisure participation, leisure satisfaction, and emotional intelligence among elementary school teachers in northern Taiwan (Tesis de Doctor of Philosophy sin publicar). The University of the Incarnate Word. Texas, United States.

Xiong, F. (2006). Constraints on leisure activities participation among Chinese Immigrants in Canada (Tesis de Doctorado en Filosofía sin publicar). Lakehead University Word, San Antonio, Canadá. 